\title{
Cognitive Defusion as Strategy to Reduce the Intensity of Craving Episodes and Improve Eating Behavior
}

\author{
Irene Hinojosa-Aguayo (D) and Felisa González (D) \\ Universidad de Granada, Spain
}

\begin{abstract}
The elaborated intrusion theory of desire proposes that craving is a cognitive motivational process involving intrusive thoughts. Changing the way we react to them, cognitive defusion (CD), should limit thought elaboration and craving. We induced chocolate craving in female chocolate cravers before $\mathrm{CD}$ (Study 1). A decrease in craving measured by a single-item scale, Visual Analogical Scale (VAS; $\left.p<.001, \eta_{\mathrm{p}}{ }^{2}=.449\right)$ and as a state, State Food Craving Questionnaire (FCQ-S; $p=.029, \eta_{\mathrm{p}}{ }^{2}=.106$ ) were found in the experimental group, while similar results were also found in group control. The reduction in craving (VAS) in group CD correlated negatively with chocolate consumption on a bogus taste test $(r=$ $-.439, p=.036)$, while the correlation was positive in the case of group control $(r=.429, p=.047)$. Food craving as a trait, measured by the Trait Food Craving Questionnaire (FCQ-T), showed negative correlations with measures of CD and mindfulness skills (lowest $r=-.313, p=.018$ ). In Study 2 participants made use of a smartphone application implementing the $\mathrm{CD}$ procedure in real contexts whenever they experienced food craving. A corresponding decline in self-reported craving was found, as well as in consumption of the craved food (indulgence) compared with the control condition. Our findings indicate that CD may be a promising intervention for tackling the elaboration of intrusive thoughts and eating behavior in young female food cravers, both in a controlled laboratory environment after a cue-food exposure craving induction procedure, as well as responding to naturally occurring food cravings in real-life settings.
\end{abstract}

Received 7 May 2021; Revised 18 November 2021; Accepted 25 November 2021

Keywords: cognitive defusion, food craving, mindfulness, smartphone application

Food craving may be defined as an intense and difficult to resist desire to consume a specific kind of food (Weingarten \& Elston, 1990) that may be a precursor of uncontrolled eating in the general population (Vainik et al., 2019). Therefore, food cravings represent an appropriate target for interventions aimed at promoting a healthy weight through improvements in eating patterns. However, conventional behavioral treatments may fail to achieve long-term weight reduction and, for some individuals, dieting might actually encourage eating problems. This has led to

Correspondence concerning this article should be addressed to Felisa González. Universidad de Granada. Departamento de Psicología Experimental. Centro de Investigación Mente, Cerebro y Comportamiento CIMCYC. Campus Cartuja, s/n. 18071 Granada (Spain). E-mail: fgreyes@ugr.es

Acknowledgments: We acknowledge M. Symonds for proofreading the manuscript and D. Cruz for his assistance in Study 1.

Funding statement: This work was supported by MINECO/FEDER, UE (Grant Number PSI2015-64345-R), and AEI/FEDER, UE (Grant Number PGC2018-096438-B-I00), awarded to F. González. Funding for open access charge: Universidad de Granada.

Conflict of interest: None. a shift away from dieting in favor of strategies centered on the actual process of eating (Schnepper et al., 2019).

For example, the elaborated intrusion (EI) theory of desire (Andrade et al., 2012; Kavanagh et al., 2005; May et al., 2012) proposes that food craving is a cognitive motivational process consisting of two distinct stages. First, food cues, as well as thoughts or memories, trigger intrusive thoughts. Second, these intrusions may be further elaborated, if for example they elicit a powerful affective reaction or a sense of deficit (Tapper, 2018), with vivid mental imagery (Schumacher et al., 2018), so that this cognitive elaboration is then experienced as craving. Therefore, the EI theory considers that craving is mainly a working memory process in which affective-

How to cite this article:

Hinojosa-Aguayo, I., \& González, F. (2022). Cognitive defusion as strategy to reduce the intensity of craving episodes and improve eating behavior. The Spanish Journal of Psychology, 25. e1. Doi:10.1017/SJP.2021.47 
laden sensory images are the object of further elaboration using internal or external information (SkorkaBrown et al., 2014). Elaboration fosters the growth of craving and the development of negative affective states that further fuel intrusions, giving rise to a cycle of intrusions and elaborations that are usually alleviated by eating the craved food (Schumacher et al., 2018).

\section{Cognitive Defusion}

Acceptance-based interventions (May et al., 2012) tackle intrusive thoughts or memories, changing how people react to them before they become elaborated. Mindfulness-based interventions have been used to treat cravings, although their effectiveness is often difficult to assess because such interventions often comprise mindfulness and non-mindfulness components (Tapper, 2018). In the present work, we selected one of the three key mindfulness components: Cognitive defusionalso referred to as decentering (Bernstein et al., 2015) or disidentification (Lacaille et al., 2014); hereafter, these labels may be considered as interchangeable in this context; however, we will use the term according to the one used in the referenced works-the other two mindfulness components are 'present moment awareness' and 'acceptance' (Tapper, 2018).

Cognitive defusion has been considered a core process of Acceptance and Commitment Therapy, where the objective is not to modify the content of thoughts but rather the way people react to them by helping individuals to separate themselves from their thoughts and emotions (Moffitt et al., 2012; for a review of the effects of mindfulness on craving and underlying mechanisms, see Tapper, 2018). In decentering, individuals are instructed to see their thoughts and feelings as transient events that are not part of themselves and that may not be a truthful reflection of reality (Tapper \& Turner, 2018). In doing so, events become less believable and, as a consequence, they have a reduced capacity to trigger desire. In this vein, the Desire Thinking theory (Caselli \& Spada, 2010) states that metacognitions play a role in the cycle of desire thinking, leading to stronger cravings, and considers the process of verbal perseveration (continual self-talk engaging the desired target) to be a component of the craving experience. Decentering could therefore represent a useful strategy for targeting these metacognitions.

\section{The Present Work}

In the present work, we examined the effectiveness of cognitive defusion in food cravers. We selected young female participants because women reportedly tend to experience stronger cravings than men (e.g., Lafay et al., 2001; Vallis, 2019; Weingarten \& Elston, 1991) and this might be particularly true in young women (Skorka-
Brown et al., 2015). In Study 1, we used a craving induction procedure involving actual exposure to chocolate before measuring self-reported craving and hunger, traits of food craving, and mindfulness skills, as well as objective measures of consumption in a covert way (bogus taste test) and healthy food choices (chocolate vs. fruit). In an attempt to extend the results of Study 1, and conduct a prelaminar feasibility assessment, in Study 2 participants used an application (app) for smartphones for a period of two weeks. The app was designed to reduce craving by following cognitive defusion instructions whenever they experienced a craving episode.

\section{Hypotheses}

If episodes of food craving are the result of intrusive thoughts prompted by external or internal food cues, which are further elaborated, we would expect to find in Study 1: $\left(H_{1 \mathrm{a}}\right)$ Training participants in cognitive defusion in order to mitigate the reactions to intrusive thoughts should lead to a reduction in self-reported craving, unhealthy-snack preference on a choice test, and food consumption on the bogus taste test, compared with a control condition; $\left(H_{1 b}\right)$ Significant positive relationships between food craving as a trait and cognitive fusion (the opposite to cognitive defusion); and $\left(H_{1 c}\right)$ Significant negative relationships between scores of mindfulness skills and both food craving as a trait and cognitive fusion (Study 1).

Regarding Study 2, participants using the app should report: $\left(H_{2 \mathrm{a}}\right)$ A greater reduction in self-reported craving; $\left(H_{2 b}\right)$ More episodes in which they had not eaten the craved food (i.e., lower indulgence); and/or $\left(H_{2 c}\right)$ : A reduction in the pattern of consumption, that is, either more reports of consuming 'less food than initially desired' or less reports of consuming 'more than initially desired', compared with a control condition.

\section{Study 1}

During craving reduction, the experimental group listened to a 3-min audio clip instructing participants to 'decenter' from their thoughts and feelings in general, under the assumption that this targets the cravingrelated reactions elicited by the induction procedure. The audio clip in the control condition substituted the cognitive defusion instructions for a reading of selected fragments of a novel (see "Materials" section). Thus, both interventions involved a verbal format that, we reasoned, may interfere with the verbal perseveration (Caselli \& Spada, 2010) of the desire thinking, but differed in the content, where only the experimental group were provided with specific instructions to target the way participants reacted to their thoughts. 
The experiment was run in two phases, with the experimental group taking part first. We incorporated measures of both formal and informal mindfulness practice, as well as measures of trait-like mindfulness facets in order to control for any a priori differences between groups that could act as confounds in the interpretation of the results. Gathering this information would also allow us to estimate the strength of the relationship between mindful-like facets, including cognitive defusion, and 'trait food craving'.

\section{Method}

\section{Participants}

Forty-five $^{1}$ female undergraduate students from the University of Granada who craved chocolate 'often' or 'always/almost every day', according to their score in the chocolate item of the Food Craving Inventory, were recruited to participate in exchange for course credits (age, $M=20.02, S D=2.55$, range 18-29). Their average body mass index (BMI) was $22.59(S D=3.57$, range 16.67-32.05), which is considered to be within the healthy weight range.

\section{Design}

To determine the effectiveness of the intervention in reducing craving, the study employed a 2 (group as between-subject factor: Cognitive defusion vs. control) x 2 (intervention as within-subject factor: Pre, post) mixed factorial design. The dependent variables were selfreported craving, food choice (healthy vs. unhealthy snack), and chocolate consumption (g) on the bogus taste test. For other analyses, the dependent variables were measures of craving, both as a state and as a trait, as well as of mindfulness skills, which are described in the next section. Power analyses were run using G*Power 3.1.

\section{Materials}

This study consisted of two phases. First, participants accessed a battery of questionnaires and sociodemographic questions (see Section "Questionnaires Completed Online before the Experimental Session") through an online survey programmed in Unipark ${ }^{2}$. Participants who met the inclusion criteria were later invited to participate in the laboratory study on subsequent days.

\footnotetext{
${ }^{1}$ An a priori power analysis for mixed design ANOVA was run, taking into account the effect size found in a previous study using a similar procedure (see Passive control study in Supplementary Materials, Point 2, at Open Science Framework, https: / / osf.io/p2fv9/) which was rather large $\left(\eta_{\mathrm{p}}{ }^{2}=.183\right.$, corresponding $\left.f=0.47\right)$. With a significance level set at .05 , the estimated minimal total sample size to achieve .80 power was 30 participants.

${ }^{2}$ www.unipark.de
}

Questionnaires Completed Online before the Experimental Session

Sociodemographic questions. These included questions related to gender, age, and estimation of weight and height to calculate the approximate BMI. Participants stated what kind of chocolate they preferred: White, milk or dark. As part of a larger research project, the questionnaire contained other questions and scales not considered in the present study.

Food Craving Inventory. Spanish version FCI-SP (Jáuregui Lobera et al., 2010). FCI-SP assesses the frequency of craving in the last month using a 5-point Likert-type response scale (from 'never' to 'always/ almost every day') for 28 food items of which we used that for chocolate.

Trait Food Craving Questionnaire, Spanish version FCQT-SP (Cepeda-Benito et al., 2000). The FCQ-T-SP is a 37-item measure of food craving using a 6-point Likert scale ranging from 'never' to 'always'. The items load onto nine factors: (a) Intentions and plans to consume food; (b) anticipation of positive reinforcement; (c) anticipation of relief from negative states and feelings; (d) lack of control over eating; (e) thoughts or preoccupation with food; (f) craving as a physiological state; (g) emotions that may be experienced before or during food cravings or eating; (h) cues that may trigger food cravings; (i) guilt from cravings and/or for giving in to them.

\section{Questionnaires/VAS Completed during the}

\section{Experimental Session}

State Food Craving Questionnaire, Spanish version, FCQ-SSP (Cepeda-Benito et al., 2000). The FCQ-S-SP measures craving for food at a given moment (state) by asking participants to indicate the extent to which they agree with 15 statements using a 5-point Likert-type response scale; we used a 6-point response scale though (from 'strongly disagree' to 'strongly agree'). The items load onto five factors: (a) Intense desire to eat; (b) anticipation of positive reinforcement; (c) anticipation of relief from negative states or feelings, (d) lack of control over eating; and (e) craving as a physiological state.

Visual Analogical Scale (VAS). This consists of a slidebar in which the moving point appears just in the center of the scale that had two extremes anchored with 'totally sated' and 'totally starving', in the case of hunger, and 'none at all' and 'absolutely', in the case of the desire to eat the craved food (craving). These extremes corresponded to a continuous scoring scale from 0 to 100 (numbers were not shown to the participants).

Questions Related to Formal and Informal Mindfulness Practice. Regarding informal practice, we included five multiple-choice questions: e.g., Q1 'Have you ever formally practiced mindfulness?', Q4 'Have you ever informally practiced mindfulness?' The 
questions and the response format are available in Supplementary Materials at the Open Science Framework, $\mathrm{OSF}^{3}$.

Five Facets Mindfulness Questionnaire (FFMQ). Spanish adaptation by Cebolla et al. (2012). The FFMQ measures a trait-like tendency to be mindful in daily life. It consists of 39 items rated on a 5-point Likert scale ranging from 'never or very rarely true' to 'very often or always true', comprising five factors (Baer et al., 2008): (a) Observing (noticing or attending to internal or external experiences); (b) describing (labeling internal experiences with words); (c) acting with awareness (attending to one's activities at that moment instead of using 'automatic pilot'); (d) non-judging (taking a non-evaluative perspective of one's own thoughts and feelings); and (e) non-reacting (tendency to allow thoughts and feelings to come and go, without getting caught up in or carried away by them).

Mindfulness Attention Awareness Scale (MAAS). Spanish adaptation by Soler et al. (2012). This scale assesses differences in the frequency of conscious states (presence of attention to and awareness of what is occurring in the present) through 15 negatively worded items, according to a 6-point Likert-type response scale ranging from 'almost never' to 'almost always'.

Cognitive Fusion Questionnaire (CFQ). Spanish adaptation by Romero-Moreno et al. (2014). The CFQ is composed of 7 items measured on a 7-point Likert scale ranging from 'never true' to 'always true' that measures general cognitive fusion (the opposite to cognitive defusion), which is the tendency for behavior to be overly dominated by cognitive events as opposed to other sources of behavioral regulation (Gillanders et al., 2014). The Spanish adaptation of the CFQ shows a one-factor structure with good internal consistency, and shows negative correlations with measures of mindfulness skills (Ruiz et al., 2017).

\section{Audio Clips}

Two audio clips of approximately $3 \mathrm{~min}$ (featuring the same female voice) were specifically recorded for this study. The cognitive defusion content was a Spanish adaption of the one used by Schumacher et al. (2018). For the control condition, we selected some fragments of the beginning of the novel by Leo Tolstoy Anna Karenina. The audio clips, as well as the transcriptions (Spanish) and descriptions (English), are available at the OSF.

\section{Procedure}

Upon arrival to the laboratory, the participants gave informed consent and completed the battery of

\footnotetext{
${ }^{3}$ https: / /osf.io/p2fv9/
}

questionnaires programmed in Unipark (see "Questionnaires/VAS Completed during the Experimental Session" section). After this, the experimental session took place, which consisted of the following phases: Chocolate craving induction, cognitive defusion (or control condition), snack choice-test, and bogus taste test. The experimental session always took place in the afternoon, when people are known to experience a strong desire for tasty snacks (van Dillen \& Andrade, 2016), between 12 p.m. and 3 p.m., to facilitate compliance with two-hour fasting taking into account the usual lunch time in Spain. All procedures used in this and the following study were approved by the Ethics Committee of the University of Granada (\#71/ CEIH/2015).

\section{Fasting Check, Initial Hunger, and Craving for Chocolate Assessments}

Participants reported their initial level of hunger using a VAS, the time elapsed since their last meal (more or less than two hours). Craving was measured using the VAS at the following timepoints: Before craving induction (VAS 1), after craving induction but before the intervention (VAS 2), and after the experimental/control intervention (VAS 3). We also measured the state of craving using the FCQ-S, before craving induction (FCQS1), immediately before the craving VAS 1 , and before the snack-choice test (FCQS2), immediately after the craving VAS 3.

\section{Induction of Craving for Chocolate: Visual and Olfactory Sensory Analysis}

First, after completing the VAS 1 and FCQS1 measures, participants were presented with a bowl containing $100 \mathrm{~g}$ of a popular Spanish sweet snack ('Conguitos', Lacasa, Zaragoza, Spain) consisting of peanuts coated with a thick cover of chocolate of their preference (white, milk, or dark chocolate, according to their response to the online questionnaire). To induce craving, we used a sensory analysis procedure in which participants had to evaluate, without tasting, the visual and olfactory properties of the chocolate using an online questionnaire programmed as a survey in Unipark (for a similar procedure involving the analysis of chocolate bars, see Andrade et al., 2012). More detailed information of this procedure is available in Supplementary Materials, available at the OSF. Immediately after completing the sensory analysis, the chocolate was withdrawn and, unknown to the participants, weighed to check whether they had complied with the requirement of not eating the chocolate. Participants were then assessed again for the level of chocolate craving experienced using the VAS scale (VAS 2). 


\section{Craving Reduction Procedure: Cognitive Defusion Audio Clip/Control Condition}

Participants in the cognitive defusion group listened to a 3-min audio clip instructing them to sit comfortably, close their eyes, and pay attention to their breathing and to the present moment. They were invited to consider their thoughts as transient entities that are not necessarily a true reflection of reality. Participants in the control condition were also instructed to sit down and pay attention to the audio clip, but instead of receiving instructions to cognitively defuse from their thoughts, they listened to the fragments of the novel. Afterwards they complete the VAS 3 and FCQS2 measures.

\section{Snack-Choice Test}

Food choice was measured covertly by offering participants a snack to take home as a gift to thank them for their participation (for a similar procedure, see van Dillen \& Andrade, 2016). They were invited to take either a KitKat bar (41.5 g), their preferred chocolate, or a pack of dehydrated fruit snacks, approximately $20 \mathrm{~g}$ of crunchy pieces of sliced fruit (Frubis, Luis Vicente, Portugal) of three different flavors: Green apple, rocha pear, and strawberry They kept the snack, but did not consume it at that moment.

\section{Bogus Taste Test}

Finally, the participants were invited to complete a second sensory analysis procedure (flavor). The bowl containing $100 \mathrm{~g}$ of the chocolate snack was presented again. They were asked to taste the chocolate and answer a set of questions related to several sensory attributes (for a similar procedure, see Schumacher et al., 2017). Detailed information of the procedure is available in Supplementary Materials, at the OSF. They were also told that the uneaten chocolate would be thrown away for hygiene reasons, so they were free to eat it all or even take the leftovers away. Once the participant left the laboratory, the experimenter estimated the amount of chocolate consumed by weighing the remaining chocolate. If a participant kept the remains of the chocolate, they were recorded as having eaten the whole amount.

\section{Results}

Significance was determined according to an alpha level of .05. Size effects were estimated by using partial etasquared for analyses of variance (ANOVAs) and Cohen's $d$ in the case of $t$-tests for pairwise contrasts. For repeated measures (RM) ANOVAs, GreenhouseGeisser correction was applied in case of violation of the assumption of sphericity. Simple main effects were computed in factorial designs to assess predicted changes within conditions involving an interaction between factors. One-tailed tests were used to examine a priori hypotheses outlined in the Introduction regarding between-condition differences (Student's $t$-test) or relationships between variables (Pearson's correlations, $r$ coefficient with 95\% CI), and two-tailed otherwise. Statistical analyses were conducted using JASP software $^{4}$.

\section{Discarding a priori Between-Group Differences}

We checked for between-group differences in the following variables: Age, BMI, hunger and craving levels at the beginning of the experiment, and scores on the FCQ-T (mean scores in FCQ-T, according to craving factor and group, are displayed in Table 1; the file 'Tables', containing all of them, is available at OSF.

The groups did not differ in most of these variables, $t_{\mathrm{s}}$ $(43)<1, p_{s}>.343$, with the exception of BMI, which was significantly higher in group control $(M=23.94, S D=$ $3.41)$ than in cognitive defusion $(M=21.30, S D=3.28)$, $t(43)=2.64, p=.011, d=0.80$, although both means fell within the range of conventional values corresponding to a healthy weight.

No differences were found between groups in any of the five factors of the FFMQ, largest $t(43)=1.36, p=.181$, or the MAAS scores, $t<1, p=.807$. There were also no group differences in CFQ scores, Welch's correction,

Table 1. Study 1. FCQ-T descriptive statistics according to group.

\begin{tabular}{llrr}
\hline & Group & Mean & SD \\
\hline \multirow{2}{*}{ Plans } & Defusion & 21.22 & 5.26 \\
& Control & 16.45 & 5.29 \\
Nos Reinf & Defusion & 17.48 & 4.23 \\
& Control & 17.81 & 4.61 \\
Control & Defusion & 10.09 & 3.20 \\
& Control & 10.45 & 4.02 \\
Thoughts & Defusion & 20.91 & 7.36 \\
& Control & 18.36 & 6.09 \\
Phys state & Defusion & 12.91 & 4.24 \\
& Control & 10.73 & 4.36 \\
Emotions & Defusion & 16.43 & 4.63 \\
& Control & 15.82 & 3.51 \\
Cues & Defusion & 7.22 & 2.41 \\
& Control & 7.50 & 2.79 \\
Guilt & Defusion & 15.87 & 4.48 \\
& Control & 15.04 & 4.52 \\
& Defusion & 8.69 & 3.91 \\
& Control & 9.68 & 4.20 \\
\hline
\end{tabular}

\footnotetext{
${ }^{4}$ https: / /jasp-stats.org
} 
$t(34.566)=0.879, p=.386$ (mean scores for each scale and factor, according to group, are depicted in Table 2)

There were no differences either between groups in formal, $\chi^{2}(2)=1.11, p=.574$, as well of informal, $\chi^{2}(2)=$ $0.43, p=.807$, mindfulness practice (for more details, see Supplementary Materials, at the OSF).

\section{Exploratory Analyses: Relationships between Mindfulness Skills, Cognitive Fusion, and Craving for Food as a Trait}

Mindfulness skills. The FCQ-T score showed negative correlations with two factors of the FFMQ, 'acting', $r=$ $-.313,95 \%$ CI [-1.000, -.069], $p=.018$, and 'non-judging', $r=-.334,95 \%$ CI $[-1.000,-.093], p=.012$, (both close to the $p$-value adjustment for multiple comparisons using Bonferroni correction, $p=.01$ ), as well as for the mindfulness and awareness state measured by the MAAS, $r=-.374,95 \%$ CI $[-1.000,-.139] p=.006$.

Cognitive fusion. There was a significant positive correlation between the FCQ-T score and the measure of cognitive fusion (CFQ), the opposite to cognitive defusion, $r=.34595 \%$ CI [0.105, 1.000], $p=.010$. The CFQ score, also showed significant negative correlations with measures of mindfulness skills, including the MAAS score, $r=-.403,95 \%$ CI $[-1.000,-.171], p=$ .003 , or the FFMQ factors 'acting', $r=-.631,95 \% \mathrm{CI}$ $[-1.000,-.454], p<.001$, 'non-judging', $r=-.673,95 \% \mathrm{CI}$ $[-1.000,-.510], p<.001$, and 'non-reacting', $r=-.387$, $95 \%$ CI $[-1.000,-.154], p=.004$ (Bonferroni corrected $p$-value for multiple comparisons, $p=.01$.).

Table 2. Study 1. Mindfulness skills (FFMQ and MASS) and cognitive fusion (CFQ) descriptive statistics according to group.

\begin{tabular}{lllr}
\hline & Group & Mean & SD \\
\hline FFMQ & & & \\
Observing & Defusion & 27.60 & 5.77 \\
& Control & 27.59 & 5.65 \\
Describing & Defusion & 26.26 & 5.95 \\
& Control & 27.27 & 4.83 \\
Acting with awareness & Defusion & 26.56 & 5.03 \\
& Control & 24.22 & 6.44 \\
Non-judging & Defusion & 24.73 & 7.92 \\
& Control & 23.86 & 7.91 \\
Non-reacting & Defusion & 20.60 & 4.53 \\
& Control & 19.31 & 3.99 \\
MAAS & Defusion & 54.87 & 12.33 \\
& Control & 54.00 & 11.33 \\
CFQ & Defusion & 25.91 & 6.88 \\
& Control & 28.36 & 11.21 \\
\hline
\end{tabular}

\section{Manipulation Check: Chocolate Craving Induction}

Self-reported craving, as measured by the VAS (VAS 1 vs. VAS 2) increased after the induction procedure, $t(44)=-3.99, p<.001, d=-0.59\left(M_{\text {pre }}=64.53, S D=\right.$ $\left.23.01 ; M_{\text {post }}=80.00, S D=18.17\right)$. Including group as a between-subjects factor in a RM-ANOVA, as a check for potential a priori differences due to the lack of randomized group assignment, showed no main effect of group or a group $x$ timepoint interaction (pre, post), $F_{s}<1$.

\section{The effect of Cognitive Defusion on Induced Craving}

The RM-ANOVA on self-reported craving (VAS) with group (cognitive defusion and control) as the betweensubject factor, and timepoint (before and after listening to the audio clip, VAS 2 vs. VAS 3 ) as the within-subject factor, yielded a main effect of timepoint, $F(1,43)=$ 34.99, $p<.001, \eta_{p}{ }^{2}=.449$ (cognitive defusion: $M_{\text {pre }}=$ $79.65, S D=19.61, M_{\text {post }}=, 63.30, S D=23.75$; control: $\left.M_{\text {pre }}=80.36, S D=17.00 ; M_{\text {post }}=68.54, S D=19.15\right)$. No other main effect or interaction was significant, largest $F<1$.

The RM-ANOVA conducted on FCQ-S scores, with group as between-subjects factor and craving factor (desire, positive reinforcement, negative affect, control, physiological state), and timepoint (pre, post intervention) as within-subject factors, yielded a main effect of timepoint, $F(1,43)=5.11, p=.029, \eta_{p}{ }^{2}=.106$, craving factor, $F(2.749,118.214)=18.22, p<.001, \eta_{p}{ }^{2}=.298$, and an interaction between these two variables, $F(4,172)=$ $2.58, p=.047, \eta_{p}{ }^{2}=.057$. No other main effect or interaction was significant, largest, $F(2.749,118.214)=2.23, p$ $=.094$. Simple main effects analysis revealed a decrease in positive reinforcement $(p=.015)$ and negative affect $(p=.007)$ after listening to the audio clips (Figure 1). Mean scores according to craving factor and timepoint are displayed in Table 3.

\section{Snack-Choice}

Choice of healthy snacks (dehydrated fruit) was codified as " 1 " and the selection of chocolate was codified as " 0 ". The percentages of participants who chose the healthy snacks were $26.09 \%$ and $9.09 \%$ in group cognitive defusion and control respectively, but this difference was not significant, $\chi^{2}(1)=2.22, p=.136$.

\section{Chocolate Consumption}

Contrary to our expectations, the groups did not differ in the amount of chocolate consumed during the bogus taste test, $t(43)<1, p=.750$, one-tailed; mean consumption for group cognitive defusion was $30.65 \mathrm{~g}(S D=$ 16.68) and for group control, this was $27.23 \mathrm{~g}(S D=$ 17.15). 


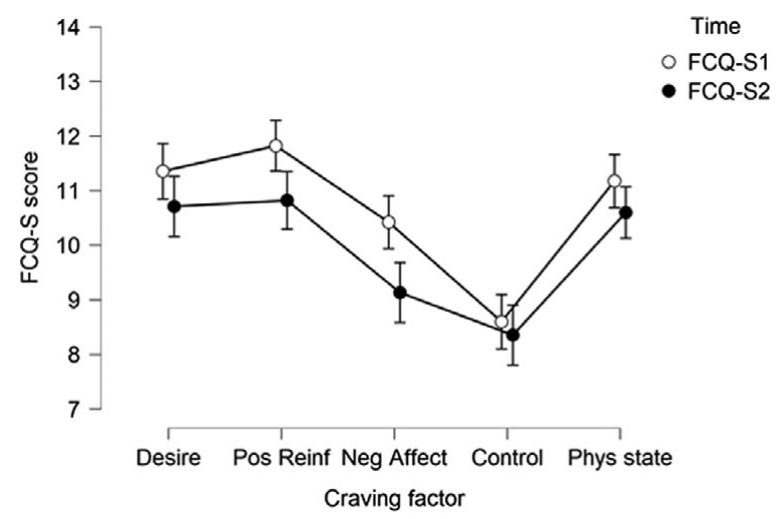

Figure 1. Study 1. Mean FCQ-S Score according to Craving Factor (Desire, Positive Reinforcement, Negative Affect, Lack of Control and Physiological State) and Timepoint Note. FCQ-S1 was administered at the beginning of the experimental session while FCQ-S2 was completed after the craving reduction procedure, immediately before the snackchoice and consumption tests. Bars represent \pm SEMs.

Table 3. Study 1. Average FCQ-S descriptive statistics according to group, factor and time: before (FCQS1) and after (FCQS2) listening to the audio clip. Des $=$ desire to eat; Pos $=$ anticipation of positive reinforcement; Neg = anticipation of relief from negative states or feelings; Con = lack of control over eating; Phys = craving as a physiological state.

\begin{tabular}{|c|c|c|c|c|}
\hline & Factor & Group & Mean & SD \\
\hline \multirow[t]{10}{*}{ FCQS1 } & \multirow[t]{2}{*}{ Des } & Defusion & 11.04 & 3.96 \\
\hline & & Control & 11.68 & 2.76 \\
\hline & \multirow[t]{2}{*}{ Pos } & Defusion & 11.69 & 3.41 \\
\hline & & Control & 11.95 & 2.78 \\
\hline & \multirow[t]{2}{*}{ Neg } & Defusion & 10.73 & 3.80 \\
\hline & & Control & 10.09 & 2.58 \\
\hline & \multirow[t]{2}{*}{ Con } & Defusion & 9.08 & 3.67 \\
\hline & & Control & 8.09 & 2.97 \\
\hline & \multirow[t]{2}{*}{ Phys } & Defusion & 10.69 & 3.59 \\
\hline & & Control & 11.68 & 2.85 \\
\hline \multirow[t]{10}{*}{ FCQS2 } & \multirow[t]{2}{*}{ Des } & Defusion & 10.43 & 4.17 \\
\hline & & Control & 11.00 & 3.16 \\
\hline & \multirow[t]{2}{*}{ Pos } & Defusion & 10.17 & 4.29 \\
\hline & & Control & 11.50 & 2.44 \\
\hline & \multirow[t]{2}{*}{ Neg } & Defusion & 8.78 & 4.39 \\
\hline & & Control & 9.50 & 2.82 \\
\hline & \multirow[t]{2}{*}{ Con } & Defusion & 8.69 & 4.01 \\
\hline & & Control & 8.00 & 3.39 \\
\hline & \multirow{2}{*}{ Phys } & Defusion & 9.78 & 3.69 \\
\hline & & Control & 11.45 & 2.24 \\
\hline
\end{tabular}

However, an exploratory analysis revealed that the decrement in self-reported craving after the intervention (VAS 2 vs. VAS 3) was negatively correlated with chocolate consumption in group cognitive defusion, $r=$ $-.439,95 \%$ CI $[-.721,-.033], p=.036$, whilst this correlation was positive in group control, $r=.429$, $95 \%$ CI $[.009, .720], p=.047$ (Figure 2). Moreover, the two correlations differed significantly according to Fisher's transformation, $z=-2.90, p=.004$.

\section{Discussion}

Listening to a 3-min audio clip that featured either the cognitive defusion instructions or the reading of a narrative, was effective in reducing self-reported craving after induction, as measured through a change in the VAS, as well as a decrease in craving as a state measured by the FCQ-S. In particular, the expectation of positive reinforcement and the reduction in negative affect, both of which might be regarded as core motivational aspects of craving related to reinforcement, were lower than at the beginning of the study. This result in the cognitive defusion group adds to the findings in the literature showing the benefits of this intervention for reducing food cravings (e.g., Forman et al., 2007; Lacaille et al., 2014; Moffitt et al., 2012; Schumacher et al., 2017, 2018) and partially confirms our first hypothesis $\left(H_{1 \mathrm{a}}\right)$.

The observation that the control condition successfully reduced self-reported craving raises doubts about the effectiveness of cognitive defusion per se. An obvious alternative explanation is that the intensity of craving declines with the mere passage of time. However, unpublished data from our laboratory (see Passive control condition, in Supplementary Materials, Point 2) suggest that this is not necessarily the case. Using distraction (playing Tetris) effectively reduced selfreported craving compared to a passive control condition (3 min waiting without performing any explicit task) in which no change of craving intensity was found. Despite the similar decline found in self-reported craving in both groups in the present study (the change in craving levels produced by the intervention after the induction measured by the VASs), this correlated negatively with chocolate consumption only in the cognitive defusion group whilst, quite unexpectedly, the opposite pattern of results was found in the control condition. Both of these issues will be taken up in the General Discussion.

The correlation patterns showed, as predicted, that craving as a trait and cognitive fusion were positively correlated $\left(H_{1 \mathrm{~b}}\right)$, and that scores on several mindfulness skills were negatively correlated with both craving as a trait and cognitive fusion $\left(H_{1 c}\right)$. These results confirm that cognitive fusion and food craving are related to each other.

Taken together, these results suggest that cognitive defusion could be a promising strategy for reducing craving, and this reduction appears to be, potentially, predictive of lower consumption of the craved food. However, participants in this study received a single 3-min intervention, which might be of limited effect. 

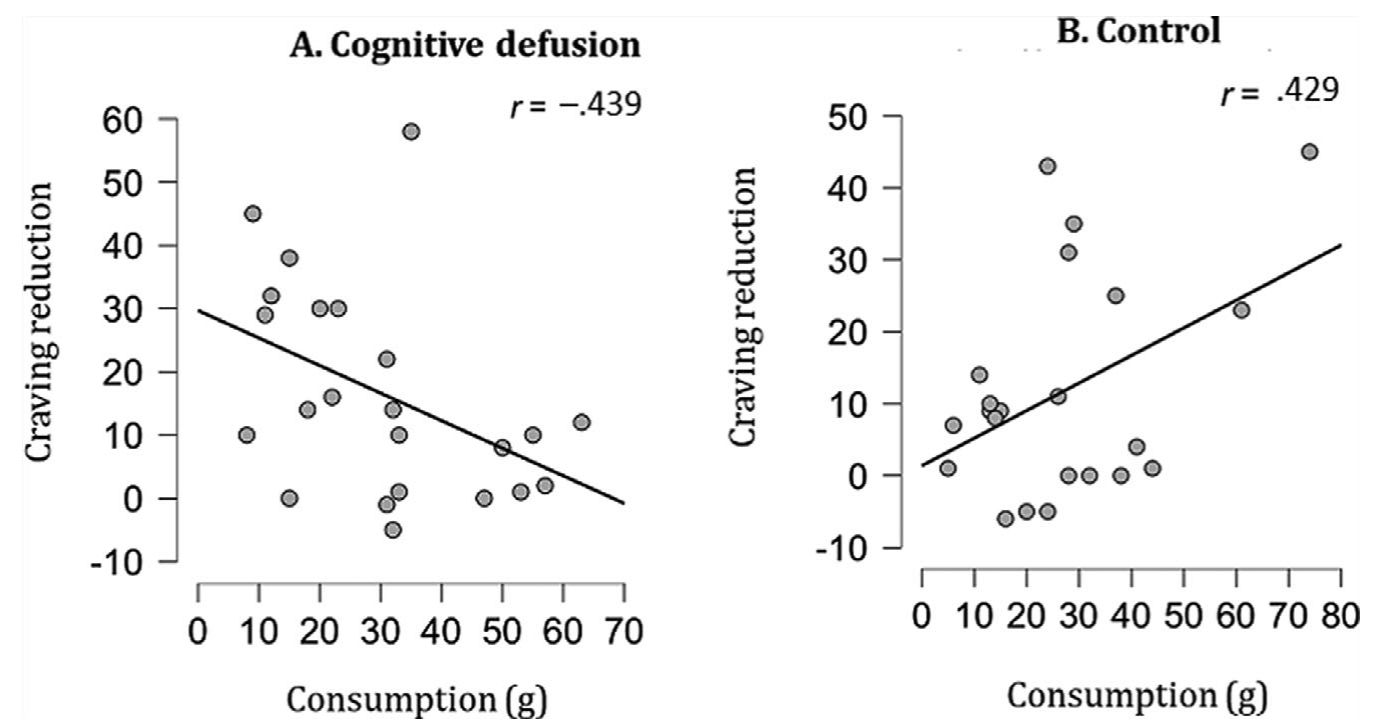

Figure 2. Study 1. Scatter Plots Showing Pearson's Coefficients for the Correlation between Craving Reduction (Measured as VAS-2 Minus VAS-3 Score) and Chocolate Consumption on the Bogus Taste Test for Groups Cognitive Defusion (A) and Control (B)

Therefore, we wondered whether the 3-min brief cognitive defusion intervention, practiced repeatedly in a real-life context whenever food craving naturally arises, will be equally effective in reducing the intensity of craving as well as in improving eating behavior.

\section{Study 2}

Therefore, to determine whether regular practice of this brief cognitive defusion experience may be effective in reducing craving and food consumption in a real context, we designed and programmed a smartphone application that could be used by the participants whenever they needed it. In this case, we recruited young female food cravers (not specifically chocolate cravers) and invited them to participate in the study for a two-week period.

\section{Method}

\section{Participants}

Forty-four young female undergraduates with a FCQ-T score above 100, who were users of an Android smartphone (Version 1.6 Donut, API level 4 or later) and met the inclusion criteria, agreed to participate in the twoweek study in exchange for course credits. Of these, 24 were then excluded due to uncompleted records or frequencies of use lower than three entries per week. The final study sample included 20 participants $^{5}(n=$ 10 in each group) with a mean age of 20.15 years $(S D=$

\footnotetext{
${ }^{5}$ An a priori power analysis for mixed design ANOVA, taking into account the effect size found in Study $1\left(\eta_{\mathrm{p}}{ }^{2}=.449\right.$, corresponding $f=$ 0.91 ) with the minimum number of entries set as a criterion (seven) as number of measures and a significance level of .05, established that the minimum total sample size to achieve .80 power was 8 .
}

2.27, range 18-26), an average BMI considered to be within the healthy weight range $(M=22.24, S D=$ 2.99 , range $=18.34-29.43$ ) and a high score on the FCQ-T, $(M=139.25, S D=29.15)$.

\section{Design}

To assess the effect of cognitive defusion on the reduction of self-reported craving we used a 2 (group as between-subject factor: Cognitive defusion vs. control) x 2 (craving measure as within-subject factor: Pre, post audio clip listening) mixed factorial design with the mean score of craving intensity across entries as the dependent variable. Other variables of interest were frequency of use, hunger level, indulgence (having eaten the craved food or not after using the app), and estimated amount eaten (if applicable) compared with the quantity initially desired before listening to the audio clip.

\section{Materials}

Participants were provided with a link to download the corresponding MIT App Inventor ${ }^{6}$ mobile application, depending on the assigned condition (cognitive defusion or control). Both versions are available at the OSF. The applications included the two 3-min audio clips used in Study 1.

\section{Procedure}

Participants were invited to visit the laboratory for an information session and were provided with written

\footnotetext{
${ }^{6}$ https: / /appinventor.mit.edu/
} 
instructions detailing how to register a new craving episode and proceed with each one. They were asked to use the app for a period of two weeks, and as often as they experienced cravings for a particular food. The procedure was as follows. They chose 'register a new craving episode' from the main menu and specified the food they craved, as well as the level of hunger and craving intensity they experienced at that moment using a slide bar anchored with 0 ('very low') and 100 ('very $\left.h i g h^{\prime}\right)$. Afterwards, they played the 3-min audio clip according to the group (cognitive defusion or control) before reporting craving intensity for a second time. Five minutes later, the app sent a notification reminding participants to enter again and choose the option 'report the last craving episode' from the main menu. They were required to report 'indulgence' (e.g., SkorkaBrown et al., 2015), specifying whether or not they finally ate the food; if they did, they had to further specify the food and estimate how much they consumed in comparison with the quantity that they initially desired before using the application (less, equal, or more).

\section{Results}

\section{Sociodemographic and Questionnaire Data}

The groups did not differ in terms of mean age or FCQ-T scores, $t_{\mathrm{s}}<1$. Further, there were no group differences in scores on any of the nine FCQ-T subscales, largest $t(18)=-1.90, p=.073$ (Table 4 displays the mean scores and standard deviations according to group and factor).

\section{Frequency of Use of the App}

Participants in both groups made use of the app with a similar frequency during the two-week period, without differences between them, $t(18)=-0.72, p=.479\left(M_{D}=\right.$ $\left.11.90, S D=3.51 ; M_{C}=13.20, S D=4.47\right)$. There were also no differences in the number of complete notifications regarding food consumption, $t(18)=-1.26, p=.223\left(M_{D}\right.$ $=9.11, S D=3.66 ; M_{C}=11.30, S D=4.11$ ).

\section{Craving Assessment}

A RM-ANOVA conducted on the mean reported craving assessments across entries, with group (cognitive defusion and control) as the between-subject factor and timepoint (pre and post audio listening) as the withinsubject factor, yielded a significant effect of timepoint, $F(1,18)=39.59, p<.001, \eta_{p}{ }^{2}=.687$ (cognitive defusion: $M_{\text {pre }}=68.39, S D=18.04, M_{\text {post }}=47.89, S D=18.92$; control: $M_{\text {pre }}=74.27, S D=10.60 ; M_{\text {post }}=57.29, S D=$ 16.01). There was no significant effect of group or a group $x$ time interaction, $F_{s}<1$.
Table 4. Study 2. FCQ-T descriptive statistics according to group.

\begin{tabular}{llrl}
\hline & Group & Mean & SD \\
\hline Plans & Defusion & 19.60 & 4.27 \\
\multirow{3}{*}{ Pos Reinf } & Control & 23.10 & 5.44 \\
& Defusion & 19.80 & 3.93 \\
Neg Affect & Control & 17.50 & 4.17 \\
& Defusion & 11.40 & 3.06 \\
Control & Control & 11.10 & 3.69 \\
& Defusion & 19.90 & 6.15 \\
Thoughts & Control & 25.30 & 7.04 \\
& Defusion & 11.00 & 3.43 \\
Phys state & Control & 12.80 & 6.23 \\
& Defusion & 18.10 & 3.72 \\
Emotions & Control & 17.60 & 3.80 \\
& Defusion & 6.50 & 2.06 \\
Cues & Control & 7.70 & 3.62 \\
\multirow{2}{*}{ Guilt } & Defusion & 16.00 & 4.32 \\
& Control & 19.30 & 3.36 \\
& Defusion & 10.60 & 4.90 \\
& Control & 11.20 & 4.07 \\
\hline
\end{tabular}

\section{Consumption of Food after the Craving Episodes}

For these analyses, we computed the frequency of indulgence by summing the number of uses of the app in which participants reported having eaten the desired food after the craving episode. Participants in group cognitive defusion reported having eaten the craved food with a significantly lower average frequency than participants in group control $t(18)=-1.82, p=.042$, onetailed, $d=-0.82\left(M_{\mathrm{D}}=4.70, S D=3.20 ; M_{\mathrm{C}}=7.40, S D=\right.$ 3.41). In addition, when they confirmed having eaten, they chose "more food than desired" with a lower average frequency than group control, $t(18)=-2.28, p=.018$, one-tailed, $d=-1.02\left(M_{\mathrm{D}}=0.60, S D=0.97 ; M_{\mathrm{C}}=1.90\right.$, $S D=1.52)$. There were no differences in the reported average frequency of the "less than desired" option, $t(18)=-1.27, p=.110$ one-tailed $\left(M_{\mathrm{D}}=1.10, S D=\right.$ $\left.1.37 ; M_{C}=2.10, S D=2.08\right)$ or "equal to desired" $t(18)=-0.22, p=.413$, one-tailed $\left(M_{\mathrm{D}}=3.00, S D=\right.$ $\left.1.83 ; M_{C}=3.20, S D=2.15\right)$.

\section{Discussion}

In both groups, listening to the 3-min audio clip produced a decrease in self-reported craving in participants using the app for a two-week period, confirming only partially our hypothesis $H_{2 a}$. This replicates the pattern of results found in Study 1, and suggests that the control condition may share some characteristics with the cognitive defusion condition, an issue that we will return to in the General Discussion.

Participants in the cognitive defusion group reported experiencing a higher number of occasions on which 
they did not eat the craved food and, when they did, the option of eating "more than desired" was chosen significantly fewer times, even if over the two-week period participants in both groups used the app with the same frequency. This pattern of results, confirming our hypothesis $H_{2 b}$ and $H_{2 c}$, respectively, is congruent with previous studies reporting that cognitive defusion may reduce the undesired impact of food craving on eating behavior without lowering the number of cravings experienced (Forman et al., 2007; Hooper et al., 2012).

\section{General Discussion}

Elaboration is at the core of the craving process, according to the EI theory (Kavanagh et al., 2005), leading to a negative affective state that ultimately motivates people to eat as a way of reducing such a state. Cognitive defusion tackles the way in which participants react to intrusive thoughts by increasing attention and awareness while considering these thoughts as transient cognitive phenomena thus preventing further elaboration. We measured self-reported craving as well as eating behavior (snack-choice and chocolate consumption) following a naturalistic induction procedure in the laboratory involving food-cue exposure (Study 1) and in reallife contexts, using a smartphone application implementing cognitive defusion to be used whenever a craving episode was experienced (Study 2).

Cognitive defusion decreased the intensity of craving measured by the VAS and also the craving state, measured by the FCQ-S. The question remains as to why these outcomes were also found in the control group. Instructing participants to attend to the narration could have prompted receptive awareness and attention to the present moment, which are two of the key mindfulness skills. Lacaille et al. (2014), reported that a non-mindful intervention, such as distraction, led to increments in awareness and acceptance to a similar extent as those produced by mindful training, but did not produce an increase in disidentification (cognitive defusion). Another potential explanation could be that in both groups, paying attention to the verbal content of the audio-clip interfered with craving-related metacognitions in the form of verbal perseveration which, according to the desire thinking theory (Caselli \& Spada, 2010), involves a continual self-talk that engages with the desired target. Cognitive defusion may have targeted these metacognitions in a more effective way than following the content of a story in the control condition, since it explicitly involves a metacognitive strategy.

Although we did not find between-group differences in chocolate consumption, which is consistent with the results of previous research (Schumacher et al., 2017), the groups differed in terms of the pattern of relationship between craving reduction measured by the VAS and the amount of chocolate consumed, being negative, as expected, in the cognitive defusion condition and positive, rather surprisingly, in the control group. Again, we can only speculate about the reasons for this discrepancy. Our participants were chocolate cravers that had been subjected to a rather strong food-exposure craving induction procedure. Without the protective effect of cognitive defusion, they might have suffered a greater craving reactivity during the bogus taste test (i.e., a rebound-like effect). Whilst awareness prompted by paying attention to the narration may have reduced craving intensity after induction, cognitive defusion might have reduced this reactivity with re-exposure to chocolate; this component was missing in the control condition.

Acceptance-based strategies, such as cognitive defusion, have been reported to engender behavioral effects even without a reduction in craving (Hooper et al., 2012), and to prompt decoupling of the relationship between craving measure and craving-related behavior (Tapper, 2018). In our study we found that food as a trait correlated positively with cognitive fusion, confirming that this could be an important target in craving-reduction strategies. Measures of awareness and acceptance (non-judging) correlated negatively both with food craving as a trait and cognitive fusion (which also correlated negatively with 'non-reacting'). This pattern of outcomes could be useful in guiding mindfulness interventions, particularly in selecting those mindfulness skills that may be more relevant to tackle food craving, although due to the reduced sample size we must interpret these findings with caution.

According to some theories (e.g., Buddhist-based), craving is thought to arise when pursuing or avoiding certain experiences, such as distress or discomfort (Lacaille et al., 2014; Moffitt et al., 2012). Therefore, cognitive defusion may be more effective in relieving distress and increasing tolerance to these experiences than strategies aimed at merely reducing the craving episodes (Forman et al., 2007). This is consistent with the reduction found in craving as a state, both in the anticipation of positive reinforcement (pleasure) and the reduction in negative affect (avoiding unpleasantness) in Study 1. Moreover, using the app during a two-week period reduced self-reported indulgence and reports of having eaten a lower amount of food when they did.

Obesity has become a pandemic, partly because we live in an obesogenic environment in which food cues generate thoughts that are immediate and automatic, giving reasons to eat more in individuals with poor executive control resources or those suffering from conditions such as stress, fatigue, or negative mood (Vallis \& Macklin, 2021). Behavioral and psychological therapies in this context are concerned with providing individuals with the skills to improve executive control in a 
person-centered manner, increasing adherence, selfefficacy, and intrinsic motivation. In this vein, previous works have showed that training in cognitive defusion (disidentification) may be more successful than training in other mindfulness skills. It might be easily taught (Lacaille et al., 2014) which may increase usability in people who are unable or unwilling to practice more formal meditation of mindfulness programs (Fisher et al., 2016). Our results add to the evidence showing that even brief cognitive defusion sessions could have potentially beneficial effects and might be implemented in smartphone applications to be used whenever is needed. The advantages of these types of procedures include their low cost, along with good accessibility and feasibility, whilst helping individuals to overcome food cravings and eating behavior by implementing a strategy built on evidence-based theories (Hsu et al., 2014).

Study 1 used quasi experimental methodology, with non-randomized healthy-student groups. However, measures were taken to examine a priori between-group differences in variables thought to be relevant for the hypotheses to be tested. In addition, the groups were specifically composed of young female participants selected for their food-craving tendencies, which could limit the generalizability of our results and impose limits on the sample sizes. Another limitation of our work could be that the manipulation used in Study 1 to reduce self-reported craving was very short. However, it is true that longer interventions are impractical in terms of time and cost. To achieve feasibility and widespread use in real-world settings, interventions must be easy to implement, with low cost, intensity, and complexity, such as those provided by applications for smartphones, allowing users to access evidencebased strategies (Chapman et al., 2018). However, due to the small size of the sample used in Study 2, it would be worthwhile to replicate these findings with a larger sample, along with a longer timeframe. Another limitation is that, for procedural reasons, we did not measure changes in mindfulness skills after the intervention in Studies 1 and 2. In particular, because these questionnaires take a long time to complete, they might have interfered with other measures in Study 1, making the application in Study 2 unmanageable, discouraging people to use it.

Food craving as a trait was found to be linked to poor awareness as well as to cognitive fusion, that is, the tendency to react to thoughts and emotions. The results of the present studies show the promising beneficial effects of practicing cognitive defusion on self-reported craving and eating behavior in young female cravers, both in the controlled environment of the laboratory as well as in real-life context using a smartphone application. These results support theories of craving such as the elaborated intrusion theory, the desire thinking theory, or Buddhist-based theories, that focus attention on the cognitive elaboration of intrusive thoughts, selftalking verbal perseveration of craving-related content, and distress-avoidance behaviors linked to poor mindfulness skills such as awareness, acceptance and, particularly, cognitive defusion.

\section{References}

Andrade, J., Pears, S., May, J., \& Kavanagh, D. J. (2012). Use of a clay modeling task to reduce chocolate craving. Appetite, 58 (3), 955-963. https: / / doi.org/10.1016/j.appet.2012.02.044

Baer, R. A., Smith, G. T., Lykins, E., Button, D., Krietemeyer, J., Sauer, S., Walsh, E., Duggan, D., \& Williams, J. M. G. (2008). Construct validity of the five facet mindfulness questionnaire in meditating and nonmeditating samples. Assessment, 15(3), 329-342. https: / / doi.org/10.1177/ 1073191107313003

Bernstein, A., Hadash, Y., Lichtash, Y., Tanay, G., Shepherd, K., \& Fresco, D. M. (2015). Decentering and related constructs: A critical review and metacognitive processes model. Perspectives on Psychological Science, 10(5), 599-617. https:/ / doi.org/10.1177/1745691615594577

Caselli, G., \& Spada, M. M. (2010). Metacognitions in desire thinking: A preliminary investigation. Behavioural and Cognitive Psychotherapy, 38(5), 629-637. https: / doi.org/ 10.1017/S1352465810000317

Cebolla, A., García-Palacios, A., Soler, J., Guillen, V., Baños, R., \& Botella, C. (2012). Psychometric properties of the Spanish validation of the five facets of mindfulness questionnaire (FFMQ). European Journal of Psychiatry, 26(2), 118-126. https:/ / doi.org/10.4321/S021361632012000200005

Cepeda-Benito, A., Gleaves, D. H., Fernández, M. C., Vila, J., Williams, T. L., \& Reynoso, J. (2000). The development and validation of Spanish versions of the State and Trait Food Cravings Questionnaires. Behaviour Research and Therapy, 38(11), 1125-1138. https: / / doi.org/10.1002/ masy.19920530125

Chapman, J., Zientara, J., \& Wilson, C. (2018). Pilot test of brief instructions to improve the self-management of general food cravings. Eating Behaviors, 30, 88-92. https:/ / doi.org/ 10.1016/j.eatbeh.2018.05.010

Fisher, N., Lattimore, P., \& Malinowski, P. (2016). Attention with a mindful attitude attenuates subjective appetitive reactions and food intake following food-cue exposure. Appetite, 99, 10-16. https:/ / doi.org/10.1016/ j.appet.2015.12.009

Forman, E. M., Hoffman, K. L., McGrath, K. B., Herbert, J. D., Brandsma, L. L., \& Lowe, M. R. (2007). A comparison of acceptance- and control-based strategies for coping with food cravings: An analog study. Behaviour Research and Therapy, 45(10), 2372-2386. https: / / doi.org/10.1016/ j.brat.2007.04.004

Gillanders, D. T., Bolderston, H., Bond, F. W., Dempster, M., Flaxman, P. E., Campbell, L., Kerr, S., Tansey, L., Noel, P., Ferenbach, C., Masley, S., Roach, L., Lloyd, J., May, L., Clarke, S., \& Remington, B. (2014). The development and 
initial validation of the Cognitive Fusion Questionnaire. Behavior Therapy, 45(1), 83-101. https:/ / doi.org/10.1016/ j.beth.2013.09.001

Hooper, N., Sandoz, E. K., Ashton, J., Clarke, A., \& McHugh, L. (2012). Comparing thought suppression and acceptance as coping techniques for food cravings. Eating Behaviors, 13(1), 62-64. https://doi.org/10.1016/j.eatbeh.2011.10.002

Hsu, A., Yang, J., Yilmaz, Y. H., Haque, M. S., Can, C., \& Blandford, A. E. (2014). Persuasive technology for overcoming food cravings and improving snack choices. In M. Jones \& P. Palanque (Eds.), CHI '14: Proceedings of the SIGCHI conference on human factors in computing systems (pp. 3403-3412). Association for Computing Machinery Press. https: / / doi.org/10.1145/2556288.2557099

Jáuregui Lobera, I., Bolaños, P., Carbonero, R., \& Valero Blanco, E. (2010). Psychometric properties of the Spanish version of Food Craving Inventory (FCI-SP). Nutrición Hospitalaria, 25(6), 984-992.

Kavanagh, D. J., Andrade, J., \& May, J. (2005). Imaginary relish and exquisite torture: The elaborated intrusion theory of desire. Psychological Review, 112, 446-467. https: / / doi.org/10.1037/0033-295X.112.2.446

Lacaille, J., Ly, J., Zacchia, N., Bourkas, S., Glaser, E., \& Knäuper, B. (2014). The effects of three mindfulness skills on chocolate cravings. Appetite, 76, 101-112. https: / / doi.org/ 10.1016/j.appet.2014.01.072

Lafay, L., Thomas, F., Mennen, L., Charles, M. A., Eschwege, E., \& Borys, J.-M. (2001). Gender differences in the relation between food cravings and mood in an adult community: Results from the Fleurbaix Laventie Ville Santé Study. International Journal of Eating Disorders, 29(2), 195-204. https: / / doi.org/10.1002/1098-108X(200103)29:2<195::AIDEAT1009>3.0.CO;2-N

May, J., Andrade, J., Kavanagh, D. J., \& Hetherington, M. (2012). Elaborated intrusion theory: A cognitive-emotional theory of food craving. Current Obesity Reports, 1(2), 114-121. https: / / doi.org/10.1007/s13679-012-0010-2

Moffitt, R., Brinkworth, G., Noakes, M., \& Mohr, P. (2012). A comparison of cognitive restructuring and cognitive defusion as strategies for resisting a craved food. Psychology and Health, 27, 74-90. https://doi.org/10.1080/ 08870446.2012 .694436

Romero-Moreno, R., Márquez-González, M., Losada, A., Gillanders, D., \& Fernández-Fernández, V. (2014). Cognitive fusion in dementia caregiving: Psychometric properties of the Spanish version of the "Cognitive Fusion Questionnaire". Behavioral Psychology/Psicología Conductual, 22(1), 117-132.

Ruiz, F. J., Suárez-Falcón, J. C., Riaño-Hernández, D., \& Gillanders, D. (2017). Psychometric properties of the Cognitive Fusion Questionnaire in Colombia. Revista Latinoamericana de Psicología, 49(1), 80-87. https: / / doi.org/ 10.1016/j.rlp.2016.09.006

Schnepper, R., Richard, A., Wilhelm, F. H., \& Blechert, J. (2019). A combined mindfulness-prolonged chewing intervention reduces body weight, food craving, and emotional eating. Journal of Consulting and Clinical Psychology, 87(1), 106-111. https:/ / doi.org/10.1037/ ccp0000361

Schumacher, S., Kemps, E., \& Tiggemann, M. (2017). Acceptance- and imagery-based strategies can reduce chocolate cravings: A test of the elaborated-intrusion theory of desire. Appetite, 113, 63-70. https:/ / doi.org/10.1016/ j.appet.2017.02.012

Schumacher, S., Kemps, E., \& Tiggemann, M. (2018). Cognitive defusion and guided imagery tasks reduce naturalistic food cravings and consumption: A field study. Appetite, 127, 393-399. https:/ / doi.org/10.1016/ j.appet.2018.05.018

Skorka-Brown, J., Andrade, J., \& May, J. (2014). Playing "Tetris" reduces the strength, frequency and vividness of naturally occurring cravings. Appetite, 76, 161-165. https:/ / doi.org/10.1016/j.appet.2014.01.073

Skorka-Brown, J., Andrade, J., Whalley, B., \& May, J. (2015). Playing Tetris decreases drug and other cravings in real world settings. Addictive Behaviors, 51, 165-170. https:/ / doi.org/10.1016/j.addbeh.2015.07.020

Soler, J., Tejedor, R., Feliu-Soler, A., Pascual, J. C., Cebolla, A., Soriano, J., Alvarez, E., \& Perez, V. (2012). Psychometric proprieties of Spanish version of Mindful Attention Awareness Scale (MAAS). Actas Españolas de Psiquiatría, 40(1), 19-26.

Tapper, K. (2018). Mindfulness and craving: Effects and mechanisms. Clinical Psychology Review, 59, 101-117. https: / doi.org/10.1016/j.cpr.2017.11.003

Tapper, K., \& Turner, A. (2018). The effect of a mindfulness-based decentering strategy on chocolate craving. Appetite, 130, 157-162. https: / / doi.org/10.1016/ j.appet.2018.08.011

Vainik, U., García-García, I., \& Dagher, A. (2019). Uncontrolled eating: A unifying heritable trait linked with obesity, overeating, personality and the brain. European Journal of Neuroscience, 50, 2430-2445. https: / / doi.org.10.1111/ ejn.14352

Vallis, M. (2019). Sustained behaviour change in healthy eating to improve obesity outcomes: It is time to abandon willpower to appreciate wanting. Clinical Obesity, 9(2), Article e12299. https:/ / doi.org/10.1111/ cob.12299

Vallis, M., \& Mackllin, D. (2021). When behaviour meets biology: If obesity is a chronic medical disease what is obesity management? Clinical Obesity, 11(3), Article e12443. https: / / doi.org/10.1111/cob.12443

van Dillen, L. F., \& Andrade, J. (2016). Derailing the streetcar named desire: Cognitive distractions reduce individual differences in cravings and unhealthy snacking in response to palatable food. Appetite, 96, 102-110. https: / / doi.org/ 10.1016/j.appet.2015.09.013

Weingarten, H. P., \& Elston, D. (1990). The phenomenology of food cravings. Appetite, 15(3), 231-246. https: / / doi.org/ 10.1016/0195-6663(90)90023-2

Weingarten, H. P., \& Elston, D. (1991). Food cravings in a college population. Appetite, 17(3), 167-175. https:/ / doi.org/10.1016/0195-6663(91)90019-O 